\title{
Bounds for the ratio of two gamma functions: from Wendel's asymptotic relation to Elezović-Giordano-Pečarić's theorem
}

Feng Qi ${ }^{1,2^{*}}$ and Qiu-Ming Luo ${ }^{3}$

"Correspondence:
qifeng618@gmail.com;
qifeng618@hotmail.com;
qifeng618@qq.com
1/nstitute of Mathematics, Henan
Polytechnic University, Jiaozuo City,
Henan Province 028043, China
${ }^{2}$ College of Mathematics, Inner
Mongolia University for
Nationalities, Tongliao City, Inner
Mongolia Autonomous Region
O28043, China
Full list of author information is
available at the end of the article

available at the end of the article

\begin{abstract}
In the expository review and survey paper dealing with bounds for the ratio of two gamma functions, along one of the main lines of bounding the ratio of two gamma functions, the authors look back and analyze some known results, including Wendel's asymptotic relation, Gurland's, Kazarinoff's, Gautschi's, Watson's, Chu's, Kershaw's, and Elezović-Giordano-Pečarić's inequalities, Lazarević-Lupaş's claim, and other monotonic and convex properties. On the other hand, the authors introduce some related advances on the topic of bounding the ratio of two gamma functions in recent years. MSC: Primary 33B15; secondary 26A48; 26A51; 26D07; 26D15; 44A10

Keywords: bound; inequality; ratio of two gamma functions; divided difference; psi function; polygamma function; completely monotonic function; Wallis' formula; inequality for sums
\end{abstract}

\section{Introduction}

Recall [1, Chapter XIII] and [2, Chapter IV] that a function $f$ is said to be completely monotonic on an interval $I$ if $f$ has derivatives of all orders on $I$ and

$$
(-1)^{n} f^{(n)}(x) \geq 0
$$

for $x \in I$ and $n \geq 0$. The celebrated Bernstein-Widder theorem [2, p.160, Theorem 12a] states that a function $f$ is completely monotonic on $[0, \infty)$ if and only if

$$
f(x)=\int_{0}^{\infty} e^{-x s} \mathrm{~d} \mu(s)
$$

where $\mu$ is a nonnegative measure on $[0, \infty)$ such that the integral (2) converges for all $x>0$. This tells us that a completely monotonic function $f$ on $[0, \infty)$ is a Laplace transform of the measure $\mu$.

It is well known that the classical Euler gamma function may be defined for $x>0$ by

$$
\Gamma(x)=\int_{0}^{\infty} t^{x-1} e^{-t} \mathrm{~d} t .
$$

The logarithmic derivative of $\Gamma(x)$, denoted by $\psi(x)=\frac{\Gamma^{\prime}(x)}{\Gamma(x)}$, is called the psi or digamma function, and $\psi^{(k)}(x)$ for $k \in \mathbb{N}$ are called the polygamma functions. It is common knowl-

\section{Springer}

@2013 Qi and Luo; licensee Springer. This is an Open Access article distributed under the terms of the Creative Commons Attribution License (http://creativecommons.org/licenses/by/2.0), which permits unrestricted use, distribution, and reproduction in any medium, provided the original work is properly cited. 
edge that the special functions $\Gamma(x), \psi(x)$, and $\psi^{(k)}(x)$ for $k \in \mathbb{N}$ are fundamental and important and have many extensive applications in mathematical sciences.

The history of bounding the ratio of two gamma functions has been longer than sixty years since the paper [3] by Wendel was published in 1948. The motivations for bounding the ratio of two gamma functions are various, including establishment of asymptotic relation, refinements of Wallis' formula, approximation to $\pi$, needs in statistics and other mathematical sciences.

In this review paper, along one of the main lines of bounding the ratio of two gamma functions, we would like to look back and analyze some known results, including Wendel's asymptotic relation, Gurland's approximation to $\pi$, Kazarinoff's refinement of Wallis' formula, Gautschi's double inequality, Watson's monotonicity, Chu's refinement of Wallis' formula, Lazarević-Lupaş's claim on monotonic and convex properties, Kershaw's first double inequality, Elezović-Giordano-Pečarićs theorem, alternative proofs of ElezovićGiordano-Pečarić's theorem and related consequences.

On the other hand, we would also like to describe some new advances in recent years on this topic, including the complete monotonicity of divided differences of the psi and polygamma functions, inequalities for sums and related results.

\section{Wendel's asymptotic relation}

Our starting point is the paper published in 1948 by Wendel, which is the earliest related one we could search out to the best of our ability.

In order to establish the classical asymptotic relation

$$
\lim _{x \rightarrow \infty} \frac{\Gamma(x+s)}{x^{s} \Gamma(x)}=1
$$

for real $s$ and $x$, by using Hölder's inequality for integrals, Wendel [3] proved elegantly the double inequality

$$
\left(\frac{x}{x+s}\right)^{1-s} \leq \frac{\Gamma(x+s)}{x^{s} \Gamma(x)} \leq 1
$$

for $0<s<1$ and $x>0$.

Wendel's original proof Let

$$
\begin{aligned}
& 0<s<1, p=\frac{1}{s}, \quad q=\frac{p}{p-1}=\frac{1}{1-s}, \\
& f(t)=e^{-s t} t^{s x}, \quad g(t)=e^{-(1-s) t} t^{(1-s) x+s-1},
\end{aligned}
$$

and apply Hölder's inequality for integrals and the recurrence formula

$$
\Gamma(x+1)=x \Gamma(x)
$$

for $x>0$ to obtain

$$
\begin{aligned}
\Gamma(x+s) & =\int_{0}^{\infty} e^{-t} t^{x+s-1} \mathrm{~d} t \leq\left(\int_{0}^{\infty} e^{-t} t^{x} \mathrm{~d} t\right)^{s}\left(\int_{0}^{\infty} e^{-t} t^{x-1} \mathrm{~d} t\right)^{1-s} \\
& =[\Gamma(x+1)]^{s}[\Gamma(x)]^{1-s}=x^{s} \Gamma(x) .
\end{aligned}
$$


Replacing $s$ by $1-s$ in (7), we get

$$
\Gamma(x+1-s) \leq x^{1-s} \Gamma(x)
$$

from which we obtain

$$
\Gamma(x+1) \leq(x+s)^{1-s} \Gamma(x+s)
$$

by substituting $x+s$ for $x$.

Combining (7) and (9), we get

$$
\frac{x}{(x+s)^{1-s}} \Gamma(x) \leq \Gamma(x+s) \leq x^{s} \Gamma(x) .
$$

Therefore, inequality (5) follows.

Letting $x$ tend to infinity in (5) yields (4) for $0<s<1$. The extension to all real $s$ is immediate on repeated application of (6).

Remark 1 Inequality (5) can be rewritten for $0<s<1$ and $x>0$ as

$$
x^{1-s} \leq \frac{\Gamma(x+1)}{\Gamma(x+s)} \leq(x+s)^{1-s}
$$

or

$$
0 \leq\left[\frac{\Gamma(x+1)}{\Gamma(x+s)}\right]^{1 /(1-s)}-x \leq s
$$

Remark 2 Using recurrence formula (6) and double inequality (10) repeatedly yields

$$
x^{1-s} \frac{\prod_{i=1}^{m}(x+i)}{\prod_{i=0}^{n-1}(x+i+s)} \leq \frac{\Gamma(x+m+1)}{\Gamma(x+n+s)} \leq(x+s)^{1-s} \frac{\prod_{i=1}^{m}(x+i)}{\prod_{i=0}^{n-1}(x+i+s)}
$$

for $x>0$ and $0<s<1$, where $m$ and $n$ are positive integers. This implies that basing on recurrence formula (6) and double inequality (10), one can bound the ratio $\frac{\Gamma(x+a)}{\Gamma(x+b)}$ for any positive numbers $x, a$ and $b$. Conversely, double inequality (12) reveals that one can also deduce corresponding bounds of the ratio $\frac{\Gamma(x+1)}{\Gamma(x+s)}$ for $x>0$ and $0<s<1$ from bounds of the ratio $\frac{\Gamma(x+a)}{\Gamma(x+b)}$ for positive numbers $x, a$, and $b$.

Remark 3 In $[4$, p.257, 6.1.46], the following limit was listed: For real numbers $a$ and $b$,

$$
\lim _{x \rightarrow \infty}\left[x^{b-a} \frac{\Gamma(x+a)}{\Gamma(x+b)}\right]=1
$$

Limits (4) and (13) are equivalent to each other since

$$
x^{t-s} \frac{\Gamma(x+s)}{\Gamma(x+t)}=\frac{\Gamma(x+s)}{x^{s} \Gamma(x)} \cdot \frac{x^{t} \Gamma(x)}{\Gamma(x+t)} .
$$

Hence, limit (13) is called Wendel's asymptotic relation in the literature. 
Remark 4 Double inequality (5) or (10) is more meaningful than limit (4) or (13), since the former implies the latter, but not conversely.

Remark 5 For more general results about the asymptotic expansion of a ratio of two gamma functions, please refer to $[5,6]$.

Remark 6 Due to unknown reasons, Wendel's paper [3] and double inequality (5) or (10) were seemingly neglected by nearly all mathematicians for more than fifty years until it was mentioned in [7], to the best of our knowledge.

Remark 7 There has been a lot of literature about asymptotic expansions of a ratio of two gamma functions. Because this expository review and survey paper is devoted to inequalities and complete monotonicity, we will not further survey the topic of asymptotic expansions of a quotient of two gamma functions. For more information, please refer to $[5,8-14]$ and closely related references therein.

\section{Gurland's double inequality}

By making use of a basic theorem in mathematical statistics concerning unbiased estimators with minimum variance, Gurland [15] presented the following inequality:

$$
\left[\frac{\Gamma((n+1) / 2)}{\Gamma(n / 2)}\right]^{2}<\frac{n^{2}}{2 n+1}
$$

for $n \in \mathbb{N}$, and so taking respectively $n=2 k$ and $n=2 k+1$ for $k \in \mathbb{N}$ in (14) yields a closer approximation to $\pi$ :

$$
\frac{4 k+3}{(2 k+1)^{2}}\left[\frac{(2 k) ! !}{(2 k-1) ! !}\right]^{2}<\pi<\frac{4}{4 k+1}\left[\frac{(2 k) ! !}{(2 k-1) ! !}\right]^{2}, \quad k \in \mathbb{N} .
$$

Remark 8 Taking respectively $n=2 k$ and $n=2 k-1$ for $k \in \mathbb{N}$ in (14) leads to

$$
\sqrt{k+\frac{1}{4}}<\frac{\Gamma(k+1)}{\Gamma(k+1 / 2)}<\frac{2 k}{\sqrt{4 k-1}}=\frac{k}{\sqrt{k-1 / 4}}, \quad k \in \mathbb{N}
$$

This is better than double inequality (10) for $x=k$ and $s=\frac{1}{2}$.

Remark 9 Double inequality (15) may be rearranged as

$$
\sqrt{k+\frac{1}{4}}<\frac{\Gamma(k+1)}{\Gamma(k+1 / 2)}<\frac{2 k+1}{\sqrt{4 k+3}}=\frac{k+1 / 2}{\sqrt{k+1 / 2+1 / 4}}, \quad k \in \mathbb{N} .
$$

It is easy to see that the upper bound in (17) is better than the corresponding one in (16). This phenomenon seemingly hints that sharper bounds for the ratio $\frac{\Gamma(k+1)}{\Gamma(k+1 / 2)}$ can be obtained only if letting $m \in \mathbb{N}$ in $n=2 m-1$ in (14). However, this is an illusion, since the 
lower bound of the following double inequality:

$$
\begin{aligned}
& \frac{k^{2}}{2 k+1} \cdot \frac{\sqrt{4(k+m)-3}}{k+m-1} \prod_{i=0}^{m-1}\left[1+\frac{1}{2(k+i)}\right] \\
& <\frac{\Gamma(k+1)}{\Gamma(k+1 / 2)}<\left(\frac{2 k}{2 k-1}\right)^{2} \frac{2(k+m)-3}{\sqrt{4(k+m)-5}} \prod_{i=0}^{m-1}\left[1-\frac{1}{2(k+i)}\right],
\end{aligned}
$$

which is derived from taking respectively $n=2(k+m-1)$ and $n=2(k+m-1)-1$ for $k \in \mathbb{N}$ in (14), is decreasing and the upper bound of it is increasing with respect to $m$. Then how can we explain the occurrence that the upper bound in (17) is stronger than the corresponding one in (16)?

Remark 10 The left-hand side inequality in (16) or (17) may be rearranged as

$$
\frac{1}{4}<\left[\frac{\Gamma(k+1)}{\Gamma(k+1 / 2)}\right]^{2}-k<\frac{1}{4}+\frac{1}{4(4 k+3)}, \quad k \in \mathbb{N} .
$$

From this, it is easier to see that inequality (14) refines double inequality (10) for $x=k$ and $s=\frac{1}{2}$.

Remark 11 It is noted that inequality (14) was recovered in [16] and extended in [17] by different approaches respectively. See Section 4 and Section 7 below.

Remark 12 Just like the paper [3], Gurland's paper [15] was ignored except that it was mentioned in $[18,19]$. The famous monograph $[20]$ recorded neither of the papers $[3,15]$.

\section{Kazarinoff's double inequality}

Starting from one form of the celebrated formula of John Wallis,

$$
\frac{1}{\sqrt{\pi(n+1 / 2)}}<\frac{(2 n-1) ! !}{(2 n) ! !}<\frac{1}{\sqrt{\pi n}}, \quad n \in \mathbb{N}
$$

which had been quoted for more than a century before 1950s by writers of textbooks, Kazarinoff proved in [17] that the sequence $\theta(n)$ defined by

$$
\frac{(2 n-1) ! !}{(2 n) ! !}=\frac{1}{\sqrt{\pi[n+\theta(n)]}}
$$

satisfies $\frac{1}{4}<\theta(n)<\frac{1}{2}$ for $n \in \mathbb{N}$. This implies

$$
\frac{1}{\sqrt{\pi(n+1 / 2)}}<\frac{(2 n-1) ! !}{(2 n) ! !}<\frac{1}{\sqrt{\pi(n+1 / 4)}}, \quad n \in \mathbb{N} .
$$

Remark 13 It was said in [17] that it is unquestionable that inequalities similar to (22) can be improved indefinitely but at a sacrifice of simplicity, which is why inequality (20) had survived so long. 
Remark 14 Kazarinoff's proof of (22) is based upon the property

$$
[\ln \phi(t)]^{\prime \prime}-\left\{[\ln \phi(t)]^{\prime}\right\}^{2}>0
$$

of the function

$$
\begin{aligned}
\phi(t) & =\int_{0}^{\pi / 2} \sin ^{t} x \mathrm{~d} x \\
& =\frac{\sqrt{\pi}}{2} \cdot \frac{\Gamma((t+1) / 2)}{\Gamma((t+2) / 2)}
\end{aligned}
$$

for $-1<t<\infty$. Inequality (23) was proved by making use of well-known Legendre's formula

$$
\psi(x)=-\gamma+\int_{0}^{1} \frac{t^{x-1}-1}{t-1} \mathrm{~d} t
$$

for $x>0$ and estimating the integrals

$$
\int_{0}^{1} \frac{x^{t}}{1+x} \mathrm{~d} x \text { and } \int_{0}^{1} \frac{x^{t} \ln x}{1+x} \mathrm{~d} x
$$

Since (23) is equivalent to the statement that the reciprocal of $\phi(t)$ has an everywhere negative second derivative, therefore, for any positive $t, \phi(t)$ is less than the harmonic mean of $\phi(t-1)$ and $\phi(t+1)$, which implies

$$
\frac{\Gamma((t+1) / 2)}{\Gamma((t+2) / 2)}<\frac{2}{\sqrt{2 t+1}}, \quad t>-\frac{1}{2} .
$$

As a subcase of this result, the right-hand side inequality in (22) is established.

Remark 15 Using recurrence formula (6) in (27) gives

$$
\left[\frac{\Gamma((t+1) / 2)}{\Gamma(t / 2)}\right]^{2}<\frac{t^{2}}{2 t+1}
$$

for $t>0$, which extends inequality (14). This shows that Kazarinoff's paper [17] contains more general conclusions and that all results in [15] stated in paper [17] contain many general conclusions, and that all results in [15] are stated below.

Replacing $t$ by $2 t$ in (27) or (28) and rearranging yield

$$
\frac{\Gamma(t+1)}{\Gamma(t+1 / 2)}>\sqrt{t+\frac{1}{4}} \Longleftrightarrow\left[\frac{\Gamma(t+1)}{\Gamma(t+1 / 2)}\right]^{2}-t>\frac{1}{4}
$$

for $t>0$, which extends the left-hand side inequality in (16) and (17). Replacing $t$ by $2 t-1$ in (27) or (28) produces

$$
\frac{\Gamma(t+1)}{\Gamma(t+1 / 2)}<\frac{2 t}{\sqrt{4 t-1}}
$$


for $t>\frac{1}{2}$, which extends the right-hand side inequality in (16). Replacing $t$ by $2 t+1$ in (27) or (28) and rearranging gives

$$
\frac{\Gamma(t+1)}{\Gamma(t+1 / 2)}<\frac{2 t+1}{\sqrt{4 t+3}}
$$

for $t>-\frac{1}{2}$, which extends the right-hand side inequality in (17).

Remark 16 By the well-known Wallis' cosine formula [21], the sequence $\theta(n)$ defined by (21) may be rearranged as

$$
\theta(n)=\frac{1}{\pi}\left[\frac{(2 n) ! !}{(2 n-1) ! !}\right]^{2}-n=\left[\frac{\Gamma(n+1)}{\Gamma(n+1 / 2)}\right]^{2}-n
$$

for $n \in \mathbb{N}$. Then inequality (22) is equivalent to

$$
\frac{1}{4}<\left[\frac{\Gamma(n+1)}{\Gamma(n+1 / 2)}\right]^{2}-n<\frac{1}{2}, \quad n \in \mathbb{N}
$$

Remark 17 Inequality (23) may be rewritten as

$$
\psi^{\prime}\left(\frac{t+1}{2}\right)-\psi^{\prime}\left(\frac{t+2}{2}\right)>\left[\psi\left(\frac{t+1}{2}\right)-\psi\left(\frac{t+2}{2}\right)\right]^{2}
$$

for $t>-1$. Letting $u=\frac{t+1}{2}$ in the above inequality yields

$$
\psi^{\prime}(u)-\psi^{\prime}\left(u+\frac{1}{2}\right)>\left[\psi(u)-\psi\left(u+\frac{1}{2}\right)\right]^{2}
$$

for $u>0$. This inequality has been generalized in [22] to the complete monotonicity of a function involving divided differences of the digamma and trigamma functions as follows.

Theorem 1 [22] For real numbers $s, t, \alpha=\min \{s, t\}$, and $\lambda$, let

$$
\Delta_{s, t ; \lambda}(x)= \begin{cases}{\left[\frac{\psi(x+t)-\psi(x+s)}{t-s}\right]^{2}+\lambda \frac{\psi^{\prime}(x+t)-\psi^{\prime}(x+s)}{t-s},} & s \neq t, \\ {\left[\psi^{\prime}(x+s)\right]^{2}+\lambda \psi^{\prime \prime}(x+s),} & s=t\end{cases}
$$

on $(-\alpha, \infty)$. Then the function $\Delta_{s, t ; \lambda}(x)$ has the following complete monotonicity:

1. For $0<|t-s|<1$,

(a) the function $\Delta_{s, t ; \lambda}(x)$ is completely monotonic on $(-\alpha, \infty)$ if and only if $\lambda \leq 1$,

(b) so is the function $-\Delta_{s, t ; \lambda}(x)$ if and only if $\lambda \geq \frac{1}{|t-s|}$;

2. For $|t-s|>1$,

(a) the function $\Delta_{s, t ; \lambda}(x)$ is completely monotonic on $(-\alpha, \infty)$ if and only if $\lambda \leq \frac{1}{|t-s|}$,

(b) so is the function $-\Delta_{s, t ; \lambda}(x)$ if and only if $\lambda \geq 1$;

3. For $s=t$, the function $\Delta_{s, s ; \lambda}(x)$ is completely monotonic on $(-s, \infty)$ if and only if $\lambda \leq 1$;

4. For $|t-s|=1$,

(a) the function $\Delta_{s, t ; \lambda}(x)$ is completely monotonic if and only if $\lambda<1$,

(b) so is the function $-\Delta_{s, t ; \lambda}(x)$ if and only if $\lambda>1$,

(c) and $\Delta_{s, t ; 1}(x) \equiv 0$. 
Remark 18 Taking in Theorem $1 \lambda=s-t>0$ produces that the function $\frac{\Gamma(x+s)}{\Gamma(x+t)}$ on $(-t, \infty)$ is increasingly convex if $s-t>1$ and increasingly concave if $0<s-t<1$.

\section{Watson's monotonicity}

In 1959, motivated by the result in [17] mentioned in Section 4, Watson [23] observed that

$$
\begin{aligned}
\frac{1}{x} \cdot \frac{[\Gamma(x+1)]^{2}}{[\Gamma(x+1 / 2)]^{2}} & ={ }_{2} F_{1}\left(-\frac{1}{2},-\frac{1}{2} ; x ; 1\right) \\
& =1+\frac{1}{4 x}+\frac{1}{32 x(x+1)}+\sum_{r=3}^{\infty} \frac{[(-1 / 2) \cdot(1 / 2) \cdot(3 / 2) \cdot(r-3 / 2)]^{2}}{r ! x(x+1) \cdots(x+r-1)}
\end{aligned}
$$

for $x>-\frac{1}{2}$, which implies that the more general function

$$
\theta(x)=\left[\frac{\Gamma(x+1)}{\Gamma(x+1 / 2)}\right]^{2}-x
$$

for $x>-\frac{1}{2}$, whose special case is the sequence $\theta(n)$ for $n \in \mathbb{N}$ defined in (21) or (32), is decreasing and

$$
\lim _{x \rightarrow \infty} \theta(x)=\frac{1}{4} \quad \text { and } \quad \lim _{x \rightarrow(-1 / 2)^{+}} \theta(x)=\frac{1}{2} .
$$

This apparently implies the sharp inequalities

$$
\frac{1}{4}<\theta(x)<\frac{1}{2}
$$

for $x>-\frac{1}{2}$,

$$
\sqrt{x+\frac{1}{4}}<\frac{\Gamma(x+1)}{\Gamma(x+1 / 2)} \leq \sqrt{x+\frac{1}{4}+\left[\frac{\Gamma(3 / 4)}{\Gamma(1 / 4)}\right]^{2}}=\sqrt{x+0.36423 \ldots}
$$

for $x \geq-\frac{1}{4}$, and, by Wallis' cosine formula [21],

$$
\frac{1}{\sqrt{\pi(n+4 / \pi-1)}} \leq \frac{(2 n-1) ! !}{(2 n) ! !}<\frac{1}{\sqrt{\pi(n+1 / 4)}}, \quad n \in \mathbb{N} .
$$

In [23], an alternative proof of double inequality (40) was also provided as follows. Let

$$
f(x)=\frac{2}{\sqrt{\pi}} \int_{0}^{\pi / 2} \cos ^{2 x} t \mathrm{~d} t=\frac{2}{\sqrt{\pi}} \int_{0}^{\infty} \exp \left(-x t^{2}\right) \frac{t \exp \left(-t^{2} / 2\right)}{\sqrt{1-\exp \left(-t^{2}\right)}} \mathrm{d} t
$$

for $x>\frac{1}{2}$. By using the fairly obvious inequalities

$$
\sqrt{1-\exp \left(-t^{2}\right)} \leq t
$$

and

$$
\frac{t \exp \left(-t^{2} / 4\right)}{\sqrt{1-\exp \left(-t^{2}\right)}}=\frac{t}{\sqrt{2 \sinh \left(t^{2} / 2\right)}} \leq 1
$$


we have, for $x>-\frac{1}{4}$,

$$
\frac{1}{\sqrt{\pi}} \int_{0}^{\infty} \exp \left(-(x+1 / 2) t^{2}\right) \mathrm{d} t<f(x)<\frac{1}{\sqrt{\pi}} \int_{0}^{\infty} \exp \left(-(x+1 / 4) t^{2}\right) \mathrm{d} t,
$$

that is to say,

$$
\frac{1}{\sqrt{x+1 / 2}}<f(x)<\frac{1}{\sqrt{x+1 / 4}} .
$$

Remark 19 It is easy to see that inequality (41) extends and improves inequalities (10), (57), and (58) if $s=\frac{1}{2}$.

Remark 20 The left-hand side inequality in (42) is better than the corresponding one in (22) but worse than the corresponding one in (15) for $n \geq 2$.

Remark 21 Formula (37) implies the complete monotonicity of the function $\theta(x)$ defined by $(38)$ on $\left(-\frac{1}{2}, \infty\right)$.

\section{Gautschi's double inequalities}

The main aim of the paper [24] was to establish the double inequality

$$
\frac{\left(x^{p}+2\right)^{1 / p}-x}{2}<e^{x^{p}} \int_{x}^{\infty} e^{-t^{p}} \mathrm{~d} t \leq c_{p}\left[\left(x^{p}+\frac{1}{c_{p}}\right)^{1 / p}-x\right]
$$

for $x \geq 0$ and $p>1$, where

$$
c_{p}=\left[\Gamma\left(1+\frac{1}{p}\right)\right]^{p /(p-1)}
$$

or $c_{p}=1$.

By an easy transformation, inequality (47) was written in terms of the complementary gamma function

$$
\Gamma(a, x)=\int_{x}^{\infty} e^{-t} t^{a-1} \mathrm{~d} t
$$

as

$$
\frac{p\left[(x+2)^{1 / p}-x^{1 / p}\right]}{2}<e^{x} \Gamma\left(\frac{1}{p}, x\right) \leq p c_{p}\left[\left(x+\frac{1}{c_{p}}\right)^{1 / p}-x^{1 / p}\right]
$$

for $x \geq 0$ and $p>1$. In particular, letting $p \rightarrow \infty$, the double inequality

$$
\frac{1}{2} \ln \left(1+\frac{2}{x}\right) \leq e^{x} E_{1}(x) \leq \ln \left(1+\frac{1}{x}\right)
$$

for the exponential integral $E_{1}(x)=\Gamma(0, x)$ for $x>0$ was derived from (50), in which the bounds exhibit the logarithmic singularity of $E_{1}(x)$ at $x=0$. 
As a direct consequence of inequality (50) for $p=\frac{1}{s}, x=0$ and $c_{p}=1$, the following simple inequality for the gamma function was deduced:

$$
2^{s-1} \leq \Gamma(1+s) \leq 1, \quad 0 \leq s \leq 1 .
$$

The second main result of the paper [24] was a sharper and more general inequality

$$
e^{(s-1) \psi(n+1)} \leq \frac{\Gamma(n+s)}{\Gamma(n+1)} \leq n^{s-1}
$$

for $0 \leq s \leq 1$ and $n \in \mathbb{N}$ than (52) by proving that the function

$$
f(s)=\frac{1}{1-s} \ln \frac{\Gamma(n+s)}{\Gamma(n+1)}
$$

is monotonically decreasing for $0 \leq s<1$. Since $\psi(n)<\ln n$, it was derived from inequality (53) that

$$
\left(\frac{1}{n+1}\right)^{1-s} \leq \frac{\Gamma(n+s)}{\Gamma(n+1)} \leq\left(\frac{1}{n}\right)^{1-s}, \quad 0 \leq s \leq 1,
$$

which was also rewritten as

$$
\frac{n !(n+1)^{s-1}}{(s+1)(s+2) \cdots(s+n-1)} \leq \Gamma(1+s) \leq \frac{(n-1) ! n^{s}}{(s+1)(s+2) \cdots(s+n-1)}
$$

and so a simple proof of Euler's product formula in the segment $0 \leq s \leq 1$ was shown by letting $n \rightarrow \infty$ in (56).

Remark 22 Double inequalities (53) and (55) can be further rearranged as

$$
n^{1-s} \leq \frac{\Gamma(n+1)}{\Gamma(n+s)} \leq \exp ((1-s) \psi(n+1))
$$

and

$$
n^{1-s} \leq \frac{\Gamma(n+1)}{\Gamma(n+s)} \leq(n+1)^{1-s}
$$

for $n \in \mathbb{N}$ and $0 \leq s \leq 1$.

Remark 23 The upper bounds in (10) and (57) have the following relationship:

$$
(n+s)^{1-s} \leq \exp ((1-s) \psi(n+1))
$$

for $0 \leq s \leq \frac{1}{2}$ and $n \in \mathbb{N}$, and inequality (59) reverses for $s>e^{1-\gamma}-1=0.52620 \ldots$, since the function

$$
Q(x)=e^{\psi(x+1)}-x
$$


was proved in $[25$, Theorem 2$]$ to be strictly decreasing on $(-1, \infty)$, with

$$
\lim _{x \rightarrow \infty} Q(x)=\frac{1}{2}
$$

This means that Wendel's double inequality (10) and Gautschi's first double inequality (57) are not included in each other but they all contain Gautschi's second double inequality (58).

Remark 24 The right-hand side inequality in (57) may be rearranged as

$$
\left[\frac{\Gamma(n+1)}{\Gamma(n+s)}\right]^{1 /(1-s)} \leq \exp (\psi(n+1)), \quad n \in \mathbb{N}
$$

This suggests us the following double inequality:

$$
\exp (\psi(\alpha(x)))<\left[\frac{\Gamma(x+t)}{\Gamma(x+s)}\right]^{1 /(t-s)} \leq \exp (\psi(\beta(x)))
$$

for real numbers $s, t$ and $x \in(-\min \{s, t\}, \infty)$, where $\alpha(x) \sim x$ and $\beta(x) \sim x$ as $x \rightarrow \infty$. For detailed information on the type of inequalities like (63), please refer to [26] and related references therein.

Remark 25 Inequality (58) can be rewritten as

$$
0 \leq\left[\frac{\Gamma(n+1)}{\Gamma(n+s)}\right]^{1 /(1-s)}-n \leq 1
$$

for $n \in \mathbb{N}$ and $0 \leq s \leq 1$.

Remark 26 In the reviews on the paper [24] by the Mathematical Reviews and the Zentralblatt MATH, there is not a word to comment on inequalities in (57) and (58). However, these two double inequalities later became a major source of a series of studies on bounding the ratio of two gamma functions.

\section{Chu's double inequality}

In 1962, by discussing that

$$
b_{n+1}(c) \gtreqless b_{n}(c)
$$

if and only if $(1-4 c) n+1-3 c \gtreqless 0$, where

$$
b_{n}(c)=\frac{(2 n-1) ! !}{(2 n) ! !} \sqrt{n+c}
$$

it was demonstrated in $[16$, Theorem 1$]$ that

$$
\frac{1}{\sqrt{\pi[n+(n+1) /(4 n+3)]}}<\frac{(2 n-1) ! !}{(2 n) ! !}<\frac{1}{\sqrt{\pi(n+1 / 4)}}, \quad n \in \mathbb{N} .
$$


As an application of (67), by using $\Gamma\left(\frac{1}{2}\right)=\sqrt{\pi}$ and (6), the following double inequality

$$
\sqrt{\frac{2 n-3}{4}}<\frac{\Gamma(n / 2)}{\Gamma(n / 2-1 / 2)} \leq \sqrt{\frac{(n-1)^{2}}{2 n-1}}
$$

for positive integers $n \geq 2$ was given in [16, Theorem 2].

Remark 27 After letting $n=2 k+1$, inequality (68) becomes

$$
\sqrt{k-\frac{1}{4}}<\frac{\Gamma(k+1 / 2)}{\Gamma(k)}<\frac{k}{\sqrt{k+1 / 4}}
$$

which is the same as (16). Taking $n=2 k+2$ in (68) leads to inequalities (17) and (19).

Notice that the reasoning directions in the two papers $[15,16]$ are opposite:

$$
\frac{(2 n-1) ! !}{(2 n) ! !} \stackrel{[16]}{\Longleftrightarrow} \frac{\Gamma(n / 2)}{\Gamma(n / 2-1 / 2)} .
$$

To some extent, the results obtained by Gurland in [15] and by Chu in [16] are equivalent to each other, and they are all special cases of those obtained by Kazarinoff in [17].

Remark 28 By Wallis' cosine formula [21], sequence (66) may be rewritten as

$$
b_{n}(c)=\frac{1}{\sqrt{\pi}} \cdot \frac{\Gamma(n+1 / 2)}{\Gamma(n+1)} \sqrt{n+c} \triangleq \frac{1}{\sqrt{\pi}} B_{c}(n)
$$

for $n \in \mathbb{N}$. Therefore, Chu discussed equivalently the necessary and sufficient conditions such that the sequence $B_{c}(n)$ for $n \in \mathbb{N}$ is monotonic.

Recently, necessary and sufficient conditions for the general function

$$
H_{a, b, c}(x)=(x+c)^{b-a} \frac{\Gamma(x+a)}{\Gamma(x+b)}
$$

on $(-\rho, \infty)$, where $a, b$, and $c$ are real numbers and $\rho=\min \{a, b, c\}$, to be logarithmically completely monotonic are presented in $[27,28]$. A positive function $f$ is said to be $\operatorname{loga-}$ rithmically completely monotonic on an interval $I \subseteq \mathbb{R}$ if it has derivatives of all orders on $I$ and its $\operatorname{logarithm} \ln f$ satisfies $(-1)^{k}[\ln f(x)]^{(k)} \geq 0$ for $k \in \mathbb{N}$ on $I$, see [29-31].

\section{Lazarević-Lupaş's claim}

In 1974, among other things, the function

$$
\theta_{\alpha}(x)=\left[\frac{\Gamma(x+1)}{\Gamma(x+\alpha)}\right]^{1 /(1-\alpha)}-x
$$

on $(0, \infty)$ for $\alpha \in(0,1)$ was claimed in [32, Theorem 2] to be decreasing and convex, and so

$$
\frac{\alpha}{2}<\left[\frac{\Gamma(x+1)}{\Gamma(x+\alpha)}\right]^{1 /(1-\alpha)}-x \leq[\Gamma(\alpha)]^{1 /(1-\alpha)} .
$$


Remark 29 The proof of [32, Theorem 2] is wrong, see [33, Remark 3.3] and [34, p.240]. However, the statements in [32, Theorem 2] are correct.

\section{Kershaw's first double inequality}

In 1983, motivated by inequality (58) obtained in [24], among other things, Kershaw presented in [35] the following double inequality:

$$
\left(x+\frac{s}{2}\right)^{1-s}<\frac{\Gamma(x+1)}{\Gamma(x+s)}<\left[x-\frac{1}{2}+\left(s+\frac{1}{4}\right)^{1 / 2}\right]^{1-s}
$$

for $0<s<1$ and $x>0$. In the literature, it is called Kershaw's first double inequality for the ratio of two gamma functions.

Kershaw's prooffor (75) Define the function $g_{\beta}$ by

$$
g_{\beta}(x)=\frac{\Gamma(x+1)}{\Gamma(x+s)}(x+\beta)^{s-1}
$$

for $x>0$ and $0<s<1$, where the parameter $\beta$ is to be determined.

It is not difficult to show, with the aid of Wendel's limit (13), that

$$
\lim _{x \rightarrow \infty} g_{\beta}(x)=1 .
$$

To prove double inequality (75), define

$$
G(x)=\frac{g_{\beta}(x)}{g_{\beta}(x+1)}=\frac{x+s}{x+1}\left(\frac{x+\beta+1}{x+\beta}\right)^{1-s},
$$

from which it follows that

$$
\frac{G^{\prime}(x)}{G(x)}=\frac{(1-s)\left[\left(\beta^{2}+\beta-s\right)+(2 \beta-s) x\right]}{(x+1)(x+s)(x+\beta)(x+\beta+1)} .
$$

This leads to

1. if $\beta=\frac{s}{2}$, then $G^{\prime}(x)<0$ for $x>0$;

2. if $\beta=-\frac{1}{2}+\left(s+\frac{1}{4}\right)^{1 / 2}$, then $G^{\prime}(x)>0$ for $x>0$.

Consequently, if $\beta=\frac{s}{2}$, then $G$ strictly decreases, and since $G(x) \rightarrow 1$ as $x \rightarrow \infty$, it follows that $G(x)>1$ for $x>0$. However, from (77), this implies that $g_{\beta}(x)>g_{\beta}(x+1)$ for $x>0$, and so $g_{\beta}(x)>g_{\beta}(x+n)$. Take the limit as $n \rightarrow \infty$ to give the result that $g_{\beta}(x)>1$, which can be rewritten as the left-hand side inequality in (75). The corresponding upper bound can be verified by a similar argument when $\beta=-\frac{1}{2}+\left(s+\frac{1}{4}\right)^{1 / 2}$, the only difference being that in this case $g_{\beta}$ strictly increases to unity.

Remark 30 The spirit of Kershaw's proof is similar to Chu's in [16, Theorem 1], as shown by (65). This idea or method was also utilized independently in [36-41] to construct, for various purposes, a number of inequalities of the type

$$
(x+\alpha)^{s-1}<\frac{\Gamma(x+s)}{\Gamma(x+1)}<(x+\beta)^{s-1}
$$

for $s>0$ and real number $x \geq 0$. 
Remark 31 It is easy to see that inequality (75) refines and extends inequalities (10) and (58).

Remark 32 Inequality (75) may be rearranged as

$$
\frac{s}{2}<\left[\frac{\Gamma(x+1)}{\Gamma(x+s)}\right]^{1 /(1-s)}-x<\left(s+\frac{1}{4}\right)^{1 / 2}-\frac{1}{2}
$$

for $x>0$ and $0<s<1$.

\section{Elezović-Giordano-Pečarić's theorem}

Inequalities (11), (19), (64), and (80), sequence (32), and functions (38) and (73) strongly suggest us to consider the monotonic and convex properties of the general function

$$
z_{s, t}(x)= \begin{cases}{\left[\frac{\Gamma(x+t)}{\Gamma(x+s)}\right]^{1 /(t-s)}-x,} & s \neq t \\ e^{\psi(x+s)}-x, & s=t\end{cases}
$$

for $x \in(-\alpha, \infty)$, where $s$ and $t$ are two real numbers and $\alpha=\min \{s, t\}$.

In 2000, Elezović, Giordano, and Pečarić gave in [34, Theorem 1] a perfect solution to the monotonic and convex properties of the function $z_{s, t}(x)$ as follows.

Theorem 2 The function $z_{s, t}(x)$ is either convex and decreasing for $|t-s|<1$ or concave and increasing for $|t-s|>1$.

Remark 33 Direct computation yields

$$
\frac{z_{s, t}^{\prime \prime}(x)}{z_{s, t}(x)+x}=\left[\frac{\psi(x+t)-\psi(x+s)}{t-s}\right]^{2}+\frac{\psi^{\prime}(x+t)-\psi^{\prime}(x+s)}{t-s} .
$$

To prove the positivity of function (82), the following formula and inequality are used as basic tools in the proof of [34, Theorem 1].

1. For $x>-1$,

$$
\psi(x+1)=-\gamma+\sum_{k=1}^{\infty}\left(\frac{1}{k}-\frac{1}{x+k}\right)
$$

2. If $a \leq b<c \leq d$, then

$$
\frac{1}{a b}+\frac{1}{c d}>\frac{1}{a c}+\frac{1}{b d}
$$

Remark 34 As consequences of Theorem 2, the following useful conclusions are derived.

1. The function

$$
e^{\psi(x+t)}-x
$$

is decreasing and convex from $(-t, \infty)$ onto $\left(t-\frac{1}{2}, t\right)$, where $t \in \mathbb{R}$. 
2. For all $x>0$,

$$
e^{\psi(x)}<\frac{1}{\psi^{\prime}(x)} \quad \text { or } \quad \psi(x)<-\ln \psi^{\prime}(x) .
$$

3. For all $x>0$ and $t>0$,

$$
\ln \left(x+t-\frac{1}{2}\right)<\psi(x+t)<\ln \left(x+e^{\psi(t)}\right)
$$

4. For $x>-\alpha$, the inequality

$$
\left[\frac{\Gamma(x+t)}{\Gamma(x+s)}\right]^{1 /(t-s)}<\frac{t-s}{\psi(x+t)-\psi(x+s)}
$$

holds if $|t-s|<1$ and reverses if $|t-s|>1$.

Remark 35 In fact, function (85) is deceasing and convex on $(-t, \infty)$ for all $t \in \mathbb{R}$. See $[25$, Theorem 2].

Remark 36 It is clear that double inequality (87) can be deduced directly from the decreasingly monotonic property of (85). Furthermore, from the decreasingly monotonic and convex properties of $(85)$ on $(-t, \infty)$, inequality $(86)$ and

$$
\psi^{\prime \prime}(x)+\left[\psi^{\prime}(x)\right]^{2}>0
$$

on $(0, \infty)$ can be derived straightforwardly.

\section{Recent advances}

Finally, we would like to state some new results related to or originating from ElezovićGiordano-Pečarić's Theorem 2 above.

\section{Alternative proofs of Elezović-Giordano-Pečarić's theorem}

The key step of verifying Theorem 2 is to prove the positivity of the right-hand side in (82), which involves divided differences of the digamma and trigamma functions. The biggest barrier or difficulty to prove the positivity of (82) is mainly how to deal with the squared term in (82).

\section{Chen's proof}

In [42], the barrier mentioned above was overcome by virtue of the well-known convolution theorem [43] for Laplace transforms, and so Theorem 2 for the special case $s+1>t>s \geq 0$ was proved. Perhaps this is the first try to provide an alternative of Theorem 2 , although it was partially successful formally.

Qi-Guo-Chen's proof

For real numbers $\alpha$ and $\beta$ with $(\alpha, \beta) \notin\{(0,1),(1,0)\}$ and $\alpha \neq \beta$, let

$$
q_{\alpha, \beta}(t)= \begin{cases}\frac{e^{-\alpha t}-e^{-\beta t}}{1-e^{-t}}, & t \neq 0 \\ \beta-\alpha, & t=0 .\end{cases}
$$


In $[44,45]$, by making use of the convolution theorem for the Laplace transform and the logarithmically convex properties of the function $q_{\alpha, \beta}(x)$ on $(0, \infty)$, an alternative proof of Theorem 2 was supplied.

Guo-Qi's first proof

In $[46,47]$, by considering monotonic properties of the function

$$
Q_{s, t ; \lambda}(u)=q_{\alpha, \beta}(u) q_{\alpha, \beta}(\lambda-u), \quad \lambda \in \mathbb{R}
$$

and still employing the convolution theorem for the Laplace transform, Theorem 2 was completely verified again.

Remark 37 For more information on the function $q_{\alpha, \beta}(t)$ and its applications, please refer to $[26,27,46-51]$ and related references therein.

\section{Guo-Qi's second proof}

In [52-55], the complete monotonic properties of the function on the right-hand side of (82) were established as follows.

Theorem 3 Let $s$ and $t$ be two real numbers and $\alpha=\min \{s, t\}$. Define

$$
\Delta_{s, t}(x)= \begin{cases}{\left[\frac{\psi(x+t)-\psi(x+s)}{t-s}\right]^{2}+\frac{\psi^{\prime}(x+t)-\psi^{\prime}(x+s)}{t-s},} & s \neq t, \\ {\left[\psi^{\prime}(x+s)\right]^{2}+\psi^{\prime \prime}(x+s),} & s=t\end{cases}
$$

on $x \in(-\alpha, \infty)$. Then the functions $\Delta_{s, t}(x)$ for $|t-s|<1$ and $-\Delta_{s, t}(x)$ for $|t-s|>1$ are completely monotonic on $x \in(-\alpha, \infty)$.

Since the complete monotonicity of the functions $\Delta_{s, t}(x)$ and $-\Delta_{s, t}(x)$ mean the positivity and negativity of the function $\Delta_{s, t}(x)$, an alternative proof of Theorem 2 was provided once again.

One of the key tools or ideas used in the proofs of Theorem 3 is the following simple but specially successful conclusion: If $f(x)$ is a function defined on an infinite interval $I \subseteq \mathbb{R}$ and it satisfies $\lim _{x \rightarrow \infty} f(x)=\delta$ and $f(x)-f(x+\varepsilon)>0$ for $x \in I$ and some fixed number $\varepsilon>0$, then $f(x)>\delta$ on $I$.

It is clear that Theorem 3 is a generalization of inequality (89).

\section{Complete monotonicity of divided differences}

In order to prove Theorem 3 , the following complete monotonic properties of a function related to a divided difference of the psi function were discovered in [54], the preprint of [55].

Theorem 4 Let $s$ and $t$ be two real numbers and $\alpha=\min \{s, t\}$. Define

$$
\delta_{s, t}(x)= \begin{cases}\frac{\psi(x+t)-\psi(x+s)}{t-s}-\frac{2 x+s+t+1}{2(x+s)(x+t)}, & s \neq t, \\ \psi^{\prime}(x+s)-\frac{1}{x+s}-\frac{1}{2(x+s)^{2}}, & s=t\end{cases}
$$

on $x \in(-\alpha, \infty)$. Then the functions $\delta_{s, t}(x)$ for $|t-s|<1$ and $-\delta_{s, t}(x)$ for $|t-s|>1$ are completely monotonic on $x \in(-\alpha, \infty)$. 
To the best of our knowledge, the complete monotonicity of functions involving divided differences of the psi and polygamma functions were investigated first in [52-57].

\section{Inequalities for sums}

As consequences of proving Theorem 4 along different approach from [55] and its preprint [54], the following algebraic inequalities for sums were procured in $[56,57]$ accidentally.

Theorem 5 Let $k$ be a nonnegative integer and let $\theta>0$ be a constant.

$$
\text { If } a>0 \text { and } b>0 \text {, then }
$$

$$
\sum_{i=0}^{k} \frac{1}{(a+\theta)^{i+1}(b+\theta)^{k-i+1}}+\sum_{i=0}^{k} \frac{1}{a^{i+1} b^{k-i+1}}>2 \sum_{i=0}^{k} \frac{1}{(a+\theta)^{i+1} b^{k-i+1}}
$$

holds for $b-a>-\theta$ and reverses for $b-a<-\theta$.

If $a<-\theta$ and $b<-\theta$, then the inequalities

$$
\sum_{i=0}^{2 k} \frac{1}{(a+\theta)^{i+1}(b+\theta)^{2 k-i+1}}+\sum_{i=0}^{2 k} \frac{1}{a^{i+1} b^{2 k-i+1}}>2 \sum_{i=0}^{2 k} \frac{1}{(a+\theta)^{i+1} b^{2 k-i+1}}
$$

and

$$
\sum_{i=0}^{2 k+1} \frac{1}{(a+\theta)^{i+1}(b+\theta)^{2 k-i+2}}+\sum_{i=0}^{2 k+1} \frac{1}{a^{i+1} b^{2 k-i+2}}<2 \sum_{i=0}^{2 k+1} \frac{1}{(a+\theta)^{i+1} b^{2 k-i+2}}
$$

hold for $b-a>-\theta$ and reverse for $b-a<-\theta$.

If $-\theta<a<0$ and $-\theta<b<0$, then inequality (95) holds and inequality (96) is valid for $a+b+\theta>0$ and is reversed for $a+b+\theta<0$.

If $a<-\theta$ and $b>0$, then inequality (95) holds and inequality (96) is valid for $a+b+\theta>0$ and is reversed for $a+b+\theta<0$.

If $a>0$ and $b<-\theta$, then inequality (95) is reversed and inequality (96) holds for $a+b+\theta<$ 0 and reverses for $a+b+\theta>0$.

If $b=a-\theta$, then inequalities (94), (95) and (96) become equalities.

Moreover, the following equivalent relation between inequality (94) and Theorem 4 was found in $[56,57]$.

Theorem 6 Inequality (94) for positive numbers $a$ and $b$ is equivalent to Theorem 4.

\section{Recent advances}

Recently, some applications, extensions, and generalizations of Theorems 3 to 5 , and related conclusions have been investigated in several recently or immediately published manuscripts such as [58-72]. For example, Theorem 1 stated in Remark 17 was obtained in [22].

The complete monotonicity of the $q$-analogue of the function $\delta_{0,0}$ defined by (93) was researched in $[73,74]$. 
Remark 38 This article is a slightly revised version of the preprint [75] and a companion paper of the preprint [76] and the articles $[26,77]$ whose preprints are $[78,79]$, respectively.

\section{Competing interests}

The authors declare that they have no competing interests.

\section{Authors' contributions}

All authors contributed equally to the manuscript and read and approved the final manuscript.

\section{Author details}

${ }^{1}$ Institute of Mathematics, Henan Polytechnic University, Jiaozuo City, Henan Province 028043, China. ${ }^{2}$ College of Mathematics, Inner Mongolia University for Nationalities, Tongliao City, Inner Mongolia Autonomous Region 028043, China. ${ }^{3}$ Department of Mathematics, Chongqing Normal University, Chongqing City, 401331, China.

\section{Acknowledgements}

The original version of this article was reported on Thursday 24 July 2008 as a talk in the seminar held at the Research Group in Mathematical Inequalities and Applications (RGMIA), School of Computer Science and Mathematics, Victoria University, Australia, while the first author was visiting the RGMIA between March 2008 and February 2009 by the grant from the China Scholarship Council. The first author would like to express many thanks to Professors Pietro Cerone and Server S. Dragomir and other local colleagues for their invitation and hospitality throughout this period. This work was partially supported by the NNSF of China under Grant No. 11361038, by the NSF Project of Chongqing City under Grant No. CSTC2011 JJA00024, by the Research Project of Science and Technology of Chongqing Education Commission under Grant No. KJ120625, and by the Fund of Chongqing Normal University, China under Grant No. 10XLR017 and 2011XLZ07, China. The authors appreciate three anonymous referees for their valuable comments on and careful corrections to the original version of this manuscript.

Received: 27 August 2013 Accepted: 22 October 2013 Published: 19 Nov 2013

\section{References}

1. Mitrinović, DS, Pečarić, JE, Fink, AM: Classical and New Inequalities in Analysis. Kluwer Academic, Dordrecht (1993)

2. Widder, DV: The Laplace Transform. Princeton University Press, Princeton (1946)

3. Wendel, JG: Note on the gamma function. Am. Math. Mon. 55(9), 563-564 (1948). doi:10.2307/2304460

4. Abramowitz, M, Stegun, IA (eds.): Handbook of Mathematical Functions with Formulas, Graphs, and Mathematical Tables, 9th printing. National Bureau of Standards, Applied Mathematics Series, vol. 55. National Bureau of Standards, Washington (1970)

5. Frame, JS: An approximation to the quotient of gamma functions. Am. Math. Mon. 56(8), 529-535 (1949). doi:10.2307/2305527

6. Tricomi, FG, Erdélyi, A: The asymptotic expansion of a ratio of gamma functions. Pac. J. Math. 1(1), 133-142 (1951). Available online at http://projecteuclid.org/euclid.pjm/1102613160

7. Merkle, M: Representations of error terms in Jensen's and some related inequalities with applications. J. Math. Anal. Appl. 231, 76-90 (1999). doi:10.1006/jmaa.1998.6214

8. Burić, T, Elezović, N: Bernoulli polynomials and asymptotic expansions of the quotient of gamma functions. J. Comput. Appl. Math. 235(11), 3315-3331 (2011)

9. Burić, T, Elezović, N: New asymptotic expansions of the quotient of gamma functions. Integral Transforms Spec. Funct. 23(5), 355-368 (2012). doi:10.1080/10652469.2011.591393

10. Fields, JL: A note on the asymptotic expansion of a ratio of gamma functions. Proc. Edinb. Math. Soc. 15(1), 43-45 (1966)

11. Fields, JL: The uniform asymptotic expansion of a ratio of gamma functions. In: Constructive Theory of Functions (Proc. Internat. Conf. Varna, 1970) (Russian), pp. 171-176. Izdat. Bolgar. Akad. Nauk, Sofia (1972)

12. Laforgia, A, Natalini, P: On the asymptotic expansion of a ratio of gamma functions. J. Math. Anal. Appl. 389(2), 833-837 (2012)

13. Mortici, C: The quotient of gamma functions by the psi function. Comput. Appl. Math. 30(3), 627-638 (2011)

14. Olver, FWJ: On an asymptotic expansion of a ratio of gamma functions. Proc. R. Ir. Acad. A 95(1), 5-9 (1995)

15. Gurland, J: On Wallis' formula. Am. Math. Mon. 63, 643-645 (1956). doi:10.2307/2310591

16. Chu, JT: A modified Wallis product and some applications. Am. Math. Mon. 69(5), 402-404 (1962). doi:10.2307/2312135

17. Kazarinoff, DK: On Wallis' formula. Edinb. Math Notes 1956(40), 19-21 (1956)

18. Dutka, J: On some gamma function inequalities. SIAM J. Math. Anal. 16, 180-185 (1985). doi:10.1137/0516013

19. Rao Uppuluri, VR: On a stronger version of Wallis' formula. Pac. J. Math. 19(1), 183-187 (1966). Available online at http://projecteuclid.org/euclid.pjm/1102993964

20. Mitrinović, DS: Analytic Inequalities. Springer, New York (1970)

21. Weisstein, EW: Wallis Cosine Formula. From MathWorld - A Wolfram Web Resource. Available online at http://mathworld.wolfram.com/WallisFormula.html

22. Qi, F, Guo, B-N: Necessary and sufficient conditions for a function involving divided differences of the di- and tri-gamma functions to be completely monotonic. arXiv:0903.3071

23. Watson, GN: A note on gamma functions. Proc. Edinburgh Math. Soc. 11 (1958/1959), no. 2, Edinburgh Math Notes No. 42 (misprinted 41) (1959), 7-9 
24. Gautschi, W: Some elementary inequalities relating to the gamma and incomplete gamma function. J. Math. Phys. 38, 77-81 (1959/60)

25. Qi, F, Guo, B-N: Sharp inequalities for the psi function and harmonic numbers. arXiv:0902.2524

26. Qi, F: Bounds for the ratio of two gamma functions. J. Inequal. Appl. 2010, Article ID 493058 (2010). doi:10.1155/2010/493058

27. Qi, F, Guo, B-N: Wendel's and Gautschi's inequalities: refinements, extensions, and a class of logarithmically completely monotonic functions. Appl. Math. Comput. 205(1), 281-290 (2008). doi:10.1016/j.amc.2008.07.005

28. Qi, F, Guo, B-N: Wendel-Gautschi-Kershaw's inequalities and sufficient and necessary conditions that a class of functions involving ratio of gamma functions are logarithmically completely monotonic. RGMIA Res. Rep. Coll. 10(1), 2 (2007). Available online at http://rgmia.org/v10n1.php

29. Atanassov, RD, Tsoukrovski, UV: Some properties of a class of logarithmically completely monotonic functions. C. R. Acad. Bulgare Sci. 41(2), 21-23 (1988)

30. Berg, C: Integral representation of some functions related to the gamma function. Mediterr. J. Math. 1(4), 433-439 (2004). doi:10.1007/s00009-004-0022-6

31. Qi, F, Guo, B-N: Complete monotonicities of functions involving the gamma and digamma functions. RGMIA Res. Rep. Coll. 7(1), 63-72 (2004). Available online at http://rgmia.org/v7n1.php

32. Lazarević, I, Lupaş, A: Functional equations for Wallis and Gamma functions. Publ. Elektrotehn. Fak. Univ. Beograd. Ser. Electron. Telecommun. Automat 461-497, 245-251 (1974)

33. Alzer, H: Sharp bounds for the ratio of q-gamma functions. Math. Nachr. 222(1), 5-14 (2001). doi:10.1002/1522-2616(200102)222:1<5::AID-MANA5>3.3.CO;2-H

34. Elezović, N, Giordano, C, Pečarić, J: The best bounds in Gautschi's inequality. Math. Inequal. Appl. 3, $239-252$ (2000). doi:10.7153/mia-03-26

35. Kershaw, D: Some extensions of W. Gautschi's inequalities for the gamma function. Math. Comput. 41, 607-611 (1983). doi:10.2307/2007697

36. Giordano, C, Laforgia, A: Inequalities and monotonicity properties for the gamma function. J. Comput. Appl. Math. 133, 387-396 (2001). doi:10.1016/S0377-0427(00)00659-2

37. Giordano, C, Laforgia, A, Pečarić, J: Monotonicity properties for some functions involving the ratio of two gamma functions. In: Bellacicco, A, Laforgia, A (eds.) Funzioni Speciali e Applicazioni, pp. 35-42. Franco Angeli, Milano (1998)

38. Giordano, C, Laforgia, A, Pečarić, J: Unified treatment of Gautschi-Kershaw type inequalities for the gamma function. Proc. VIIIth Symposium on Orthogonal Polynomials and Their Applications (Seville, 1997). J. Comput. Appl. Math. 99(1-2), 167-175 (1998). doi:10.1016/S0377-0427(98)00154-X

39. Laforgia, A: Further inequalities for the gamma function. Math. Comput. 42(166), 597-600 (1984). doi:10.2307/2007604

40. Lorch, L: Inequalities for ultraspherical polynomials and the gamma function. J. Approx. Theory 40(2), 115-120 (1984). doi:10.1016/0021-9045(84)90020-0

41. Palumbo, B: A generalization of some inequalities for the gamma function. J. Comput. Appl. Math. 88(2), 255-268 (1998). doi:10.1016/S0377-0427(97)00187-8

42. Chen, C-P: Monotonicity and convexity for the gamma function. J. Inequal. Pure Appl. Math. 6(4), 100 (2005). Available online at http://www.emis.de/journals/JIPAM/article574.html

43. Weisstein, EW: Laplace Transform. From MathWorld - A Wolfram Web Resource. Available online at http://mathworld.wolfram.com/LaplaceTransform.html

44. Qi, F, Guo, B-N, Chen, C-P: The best bounds in Gautschi-Kershaw inequalities. Math. Inequal. Appl. 9(3), 427-436 (2006). doi:10.7153/mia-09-41

45. Qi, F, Guo, B-N, Chen, C-P: The best bounds in Gautschi-Kershaw inequalities. RGMIA Res. Rep. Coll. 8(2), 17 (2005). Available online at http://rgmia.org/v8n2.php

46. Guo, B-N, Qi, F: An alternative proof of Elezović-Giordano-Pečarić's theorem. Math. Inequal. Appl. 14(1), 73-78 (2011). doi:10.7153/mia-14-06

47. Qi, F, Guo, B-N: An alternative proof of Elezović-Giordano-Pečarić's theorem. arXiv:0903.1174

48. Guo, B-N, Qi, F: Properties and applications of a function involving exponential functions. Commun. Pure Appl. Anal. 8(4), 1231-1249 (2009). doi:10.3934/cpaa.2009.8.1231

49. Qi, F: Monotonicity and logarithmic convexity for a class of elementary functions involving the exponential function. RGMIA Res. Rep. Coll. 9(3), 3 (2006). Available online at http://rgmia.org/v9n3.php

50. Qi, F: Three-log-convexity for a class of elementary functions involving exponential function. J. Math. Anal. Approx. Theory 1(2), 100-103 (2006)

51. Qi, F, Luo, Q-M, Guo, B-N: The function $\left(b^{x}-a^{x}\right) / x$ : ratio's properties. In: Alladi, K, Milovanović, GV, Rassias, MT (eds.) Analytic Number Theory, Approximation Theory, and Special Functions, pp. 673-682. Springer, Berlin (2014)

52. Guo, B-N, Qi, F: A class of completely monotonic functions involving divided differences of the psi and tri-gamma functions and some applications. J. Korean Math. Soc. 48(3), 655-667 (2011). doi:10.4134/JKMS.2011.48.3.655

53. Qi, F: A completely monotonic function involving divided differences of psi and polygamma functions and an application. RGMIA Res. Rep. Coll. 9(4), 8 (2006). Available online at http://rgmia.org/v9n4.php

54. Qi, F: The best bounds in Kershaw's inequality and two completely monotonic functions. RGMIA Res. Rep. Coll. 9(4), 2 (2006). Available online at http://rgmia.org/v9n4.php

55. Qi, F, Guo, B-N: Completely monotonic functions involving divided differences of the di- and tri-gamma functions and some applications. Commun. Pure Appl. Anal. 8(6), 1975-1989 (2009). doi:10.3934/cpaa.2009.8.1975

56. Qi, F: A completely monotonic function involving divided difference of psi function and an equivalent inequality involving sum. RGMIA Res. Rep. Coll. 9(4), 5 (2006). Available online at http://rgmia.org/v9n4.php

57. Qi, F: A completely monotonic function involving the divided difference of the psi function and an equivalent inequality involving sums. ANZIAM J. 48(4), 523-532 (2007). doi:10.1017/S1446181100003199

58. Guo, B-N, Qi, F: A completely monotonic function involving the tri-gamma function and with degree one. Appl. Math. Comput. 218(19), 9890-9897 (2012). doi:10.1016/j.amc.2012.03.075

59. Guo, B-N, Qi, F: An extension of an inequality for ratios of gamma functions. J. Approx. Theory 163(9), 1208-1216 (2011). doi:10.1016/j.jat.2011.04.003 
60. Guo, B-N, Qi, F: Monotonicity and logarithmic convexity relating to the volume of the unit ball. Optim. Lett. 7(6), 1139-1153 (2013). doi:10.1007/s11590-012-0488-2

61. Guo, B-N, Qi, F: Monotonicity of functions connected with the gamma function and the volume of the unit ball. Integral Transforms Spec. Funct. 23(9), 701-708 (2012). doi:10.1080/10652469.2011.627511

62. Guo, B-N, Qi, F: Refinements of lower bounds for polygamma functions. Proc. Am. Math. Soc. 141(3), 1007-1015 (2013). doi:10.1090/S0002-9939-2012-11387-5

63. Guo, B-N, Zhao, J-L, Qi, F: A completely monotonic function involving the tri- and tetra-gamma functions. Math. Slovaca 63(3), 469-478 (2013). doi:10.2478/s12175-013-0109-2

64. Guo, S, Xu, J-G, Qi, F: Some exact constants for the approximation of the quantity in the Wallis' formula. J. Inequal. Appl. 2013, 67 (2013). doi:10.1186/1029-242X-2013-67

65. Qi, F: Limit formulas for ratios between derivatives of the gamma and digamma functions at their singularities. Filomat 27(4), 601-604 (2013). doi:10.2298/FIL1304601Q

66. Qi, F, Berg, C: Complete monotonicity of a difference between the exponential and trigamma functions and properties related to a modified Bessel function. Mediterr. J. Math. 10(4), 1683-1694 (2013) doi:10.1007/s00009-013-0272-2

67. Qi, F, Cerone, P, Dragomir, SS: Complete monotonicity of a function involving the divided difference of psi functions Bull. Aust. Math. Soc. 88(2), 309-319 (2013). doi:10.1017/S0004972712001025

68. Qi, F, Guo, B-N: Necessary and sufficient conditions for functions involving the tri- and tetra-gamma functions to be completely monotonic. Adv. Appl. Math. 44(1), 71-83 (2010). doi:10.1016/j.aam.2009.03.003

69. Qi, F, Luo, Q-M, Guo, B-N: Complete monotonicity of a function involving the divided difference of digamma functions. Sci. China Math. 56(11), 2315-2325 (2013). doi:10.1007/s11425-012-4562-0

70. Qi, F, Wei, C-F, Guo, B-N: Complete monotonicity of a function involving the ratio of gamma functions and applications. Banach J. Math. Anal. 6(1), 35-44 (2012)

71. Zhao, J-L, Guo, B-N, Qi, F: A refinement of a double inequality for the gamma function. Publ. Math. (Debr.) 80(3-4), 333-342 (2012). doi:10.5486/PMD.2012.5010

72. Zhao, J-L, Guo, B-N, Qi, F: Complete monotonicity of two functions involving the tri- and tetra-gamma functions. Period. Math. Hung. 65(1), 147-155 (2012). doi:10.1007/s10998-012-9562-x

73. Qi, F: A completely monotonic function related to the $q$-trigamma function. Politehn. Univ. Bucharest Sci. Bull. Ser. A Appl. Math. Phys. 76 (2014, in press)

74. Qi, F: Complete monotonicity of functions involving the $q$-trigamma and $q$-tetragamma functions. arXiv:1301.0155

75. Qi, F: Bounds for the ratio of two gamma functions-From Wendel's limit to Elezović-Giordano-Pečarić's theorem. arXiv:0902.2514

76. Qi, F: Bounds for the ratio of two gamma functions-From Gautschi's and Kershaw's inequalities to completely monotonic functions. arXiv:0904.1049

77. Qi, F, Luo, Q-M: Bounds for the ratio of two gamma functions - from Wendel's and related inequalities to logarithmically completely monotonic functions. Banach J. Math. Anal. 6(2), 132-158 (2012)

78. Qi, F: Bounds for the ratio of two gamma functions. RGMIA Res. Rep. Coll. 11(3), 1 (2008). Available online at http://rgmia.org/v11n3.php

79. Qi, F: Bounds for the ratio of two gamma functions-From Wendel's and related inequalities to logarithmically completely monotonic functions. arXiv:0904.1048

10.1186/1029-242X-2013-542

Cite this article as: Qi and Luo: Bounds for the ratio of two gamma functions: from Wendel's asymptotic relation to

Elezović-Giordano-Pečarić's theorem. Journal of Inequalities and Applications 2013, 2013:542

\section{Submit your manuscript to a SpringerOpen ${ }^{\circ}$ journal and benefit from:}

- Convenient online submission

- Rigorous peer review

- Immediate publication on acceptance

Open access: articles freely available online

- High visibility within the field

- Retaining the copyright to your article

Submit your next manuscript at $>$ springeropen.com 